REVIEW

\title{
Lemierre's syndrome: more than a historical curiosa
}

\author{
T Riordan, M Wilson
}

Postgrad Med J 2004;80:328-334. doi: 10.1136/pgmi.2003.014274

Lemierre's syndrome is a severe illness caused by the anaerobic bacterium, Fusobacterium necrophorum which typically occurs in healthy teenagers and young adults. The infection originates in the throat and spreads via a septic thrombophlebitis of the tonsillar vein and internal jugular vein. The ensuing bacteraemia is complicated by septic emboli to a range of sites such as lung, joints, and bones. Although rare, there is evidence of a resurgence in the condition in recent years, possibly associated with reduced use of antibiotic therapy for sore throats. The typical clinical picture is characteristic but many clinicians are unaware of the condition and diagnosis is offen delayed with potentially fatal consequences.

See end of article for authors' affiliations

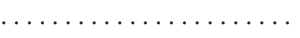

Correspondence to: Dr Terry Riordan, Microbiology Department, Royal Devon and Exeter Healthcare NHS Trust, Church Lane, Heavitree, Exeter EX2 5AD, UK; terry. riordan@rdehc-tr.swest. nhs.uk

Submitted 14 January 2003

Accepted 28 May 2003
F usobacteria are obligate anaerobic Gram negative rods that are normal flora in both animals and humans in the upper respiratory tract. One species, now known as Fusobacterium necrophorum, was recognised as an important pathogen in veterinary medicine in the late 19th century, ${ }^{1}$ causing a variety of conditions such as calf diphtheria, stomatitis of calves, lambs and pigs, foot rot in cattle and sheep, and multiple abscesses in the lungs and liver of cattle and pigs. Because of the necrotic abscesses produced by the bacterium, the condition came to be known as necrobacillosis particularly in UK publications.

The first case report of human necrobacillosis was in 1900 by Courmont and Cade. ${ }^{2}$ The term is now used to include any septicaemic infection caused by Fusobacterium necrophorum. As demonstrated by various authors including Alston, it can be associated with diverse sources of infection, including the upper respiratory tract, genitourinary tract, and the gut. ${ }^{3}$

In 1936, André Lemierre, in a discourse on anaerobic septicaemias, divided patients into six groups according to the source of infection: inflammatory lesions of the nasopharynx, particularly tonsillar/peritonsillar abscess, lesions of mouth and jaw, otitis media or mastoiditis, purulent postpartum endometritis, appendicitis, and urinary tract infections. ${ }^{4}$ Lemierre described the first group as "anaerobic postanginal septicaemias". The patients in this group were young, previously healthy, adolescents or young adults presenting with initial pharyngotonsillitis or peritonsillar abscess, often followed by swelling and tenderness along the sternomastoid muscle due to septic thrombophlebitis of the internal jugular vein. High fevers and rigors developed within a week and subsequently metastatic abscesses commonly to lung, bone, joints, and skin and soft tissues.

\begin{abstract}
"To anyone instructed as to the nature of these septicaemias it becomes relatively easy to make a diagnosis on the simple clinical findings, the appearance and repetition several days after the onset of a sore throat of severe pyrexial attacks with an initial rigor and still more certainly the occurrence of pulmonary infarcts and arthritic manifestations make a syndrome that is so characteristic that mistake is almost impossible". ${ }^{4}$
\end{abstract}

\section{NECROBACILLOSIS}

This condition has come to be known as Lemierre's syndrome, although in a manner familiar to Humpty Dumpty, these words can mean to individual authors exactly what they choose them to mean. Although there is general agreement on the central classical cases, which involve postanginal sepsis with isolation of $F$ necrophorum from blood cultures, there is a lack of consensus. Some authors include in Lemierre's syndrome cases in which the source of infection arises not from the throat but from the ears, mastoid, or indeed tooth infection. ${ }^{5}$ At the same time other authors do not require the isolation of $F$ necrophorum. For example in the series of cases reviewed from the literature by Sinave et al, only 23 out of 37 cases had $F$ necrophorum detected, the remaining 13 patients had a range of other organisms, both aerobes and anaerobes. ${ }^{6}$ It is possible that all cases originate from a $F$ necrophorum infection, but that for a variety of reasons, including prior antibiotic therapy, the organism is not detected in some patients.

The current authors take the view that inclusion of non-postanginal cases in Lemierre's syndrome is unhelpful (fig 1). This is on the basis that the age groups are quite distinct. Ear and mastoid cases usually involve preschool children, contrasting with the typical age of 1619 years of classical Lemierre's. In addition, earassociated cases do not usually result in internal jugular venous thrombophlebitis or metastatic lung lesions. By contrast meningitis occurs much more commonly in this group than in classical postanginal cases. This review will therefore focus principally on classical, that is postanginal, Lemierre's syndrome with the isolation of $F$ necrophorum from blood culture or other normally sterile site. 


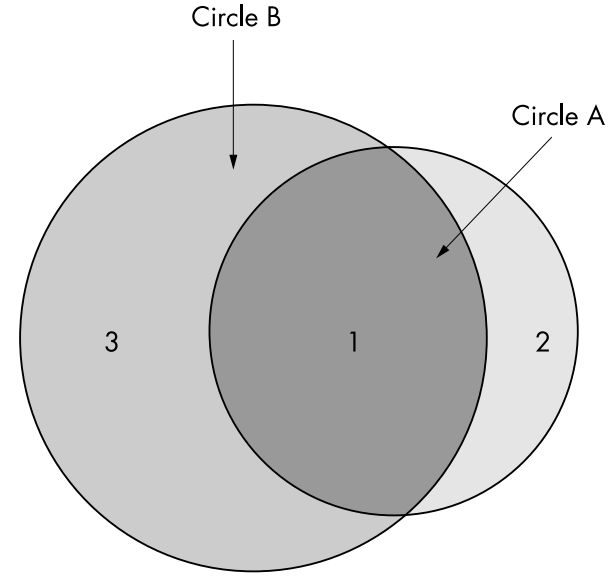

Figure 1 Patient groups: necrobacillosis and Lemierre's. Circle A = postanginal sepsis with internal jugular vein thrombosis and metatastic manifestations and circle $B=$ necrobacillosis. 1 = classical Lemierre's postangingal sepsis, 2 = clinical Lemierre's but $F$ necrophorum not detected, 3 = necrobacillosis from other source.

\section{EPIDEMIOLOGY}

Postanginal sepsis due to Lemierre's syndrome appears to have been relatively common in the preantibiotic era. In 1955 Alston identified a total of 280 cases of necrobacillosis in the world literature. ${ }^{3}$ It was sparsely reported in the 60 s and 70 s, possibly because of widespread use of penicillin for throat infection combined with relatively poor standards of anaerobic bacteriology.

Several papers in the 1980s highlighted that this "forgotten disease" had not gone away. ${ }^{58}$ Although it remains a rare disease with an incidence of approximately one per million persons per year ${ }^{5}$ there has been a remarkable resurgence of publications in the cited literature since 1990. Recent papers have suggested that the incidence of the condition is rising. ${ }^{5-11}$ Published UK surveillance 1990-2000 data showed a peak of cases in 1999. ${ }^{12}$ Subsequent reference laboratory data suggest that this rise has continued in 2001 and 2002 (J Brazier, personal communication). One hypothesis is that primary care physicians are now heavily discouraged from prescribing antibiotics for sore throats ${ }^{13}$ and that early infections which would previously have been aborted by antibiotics now progress to the full blown syndrome. Another potential factor is that $F$ necrophorum isolates are now reported to be erythromycin resistant. ${ }^{12}$ However it is erroneous to imply that antibiotic therapy before admission can reliably interrupt the natural history. Thus in Hagelskjaer's series $53 \%$ of patients had received antibiotics before admission. The putative rise of $F$ necrophorum bacteraemia has also been postulated to be due to relative improvements in anaerobic blood culture techniques resulting in improved detection.

\section{PATHOGENESIS}

Lemierre's syndrome almost invariably arises in patients who were previously fit. This is in striking contrast to necrobacillosis arising outside the head and neck. In Hagelskjaer's series of non-head and neck cases, ${ }^{5}$ nine out of 25 had an underlying cancer, seven were alcohol or drug abusers, and four had insulin dependent diabetes mellitus.

Given that $F$ necrophorum is found in the normal flora of the oropharynx there must be factors that precipitate invasive infection. Mucosal damage by bacterial or viral pharyngitis may be a precipitating factor. ${ }^{10}$ Several reports have described infected patients to have serological evidence of recent EpsteinBarr virus infection. ${ }^{14}$ This may induce immunosuppression with a transient decrease in $\mathrm{T}$ cell mediated immunity facilitating secondary bacterial infection.

Pathogenic mechanisms are complex and various toxins have been identified. Unlike other anaerobic bacteria, $F$ necrophorum possesses a lipopolysaccharide endotoxin that has been shown to be lethal in animal models. ${ }^{6}$ Robert showed that the inflammatory response in $F$ necrophorum infections is largely dependent on production of an extracellular leucocidin which is relatively heat stable. ${ }^{15} F$ necrophorum aggregates human platelets in vitro without lysing them. The resulting intravasular coagulation may contribute to the creation of an anaerobic environment and is probably a key virulence factor in generating a septic thrombophlebitis in the tonsillar veins, which propagates centrally to involve the internal jugular vein, whence septic emboli are disseminated to the characteristic sites of metastatic abscesses.

Another possible factor is that infection may follow recent acquisition of a virulent strain. Most isolates of fusobacterium from patients with Lemierre's disease are identified as $F$ necrophorum subspecies necrophorum. Only this subspecies aggregates human platelets. Subspecies necrophorum also produces more leucocidin and endotoxin than other subspecies. ${ }^{10}$

$F$ necrophorum is often found mixed with other pathogens, for example $33 \%$ of patients with Lemierre's syndrome in Hagelskjaer's study had polymicrobial infections. ${ }^{5}$ There may be synergy with other anaerobic or microaerophilic bacteria, which will lower the oxygen concentration and thus provide anaerobic conditions to aid growth within the abscess. ${ }^{16}$ However, given the fact that the majority of cases involve single organism bacteraemia, this is likely only to be relevant in the initiation of infection in the throat.

\section{CLINICAL FEATURES}

\section{Onset}

The onset of the septicaemic illness is heralded by a marked rise in fever to $39-41{ }^{\circ} \mathrm{C}$, often followed by a rigor. This typically occurs 4-5 days after the onset of the sore throat but the interval may be up to 12 days. ${ }^{4}$

\section{Oropharyngeal/cervical lesions}

The initial sore throat varies in severity and may indeed have started to improve when the onset of the septicaemic illness occurs. On the other hand the tonsillar lesion may be severe enough to induce dysphagia. When the patient presents to hospital the appearance of the throat can vary from a normal appearance through mild tonsillar and/or pharyngeal inflammation to a severe exudative tonsillitis with peritonsillar abscess.

"Be not deceived by a comparatively innocent appearing pharynx as the veins of the tonsil may be carrying the death sentence of your patient ${ }^{\prime \prime}{ }^{17}$

Box 1: Features of septicaemia with Lemierre's syndrome

- Respiratory failure requiring mechanical ventilation is not usual despite extent of lung involvement and severity of sepsis.

- Inotropic support is rarely required.

- Renal failure requiring haemofiltration or dialysis is exceptional.

- Mortality is around $5 \%$ in published series. 
Patients often complain of neck pain and sometimes stiffness. Cervical lymphadenopathy may be present either unilaterally or bilaterally, often in the anterior triangle. More importantly, there may be a tender (normally unilateral) swelling at the angle of the jaw or anterior to, and parallel with, the sternomastoid muscle, reflecting the development of internal jugular venous thrombophlebitis. ${ }^{18}$ This has been detected in $26 \%-45 \%$ of cases (table 1 ).

In addition to peritonsillar abscess and internal jugular venous thrombosis, additional local septic complications that may occur include parapharyngeal abscess ${ }^{20}$ and paratracheal abscess. ${ }^{21}$

\section{Pulmonary involvement}

Pulmonary involvement precipitated by septic embolisation is extremely common (table 1). Lemierre described how lung lesions manifest early (sometimes on the first day of the septicaemia) and characteristically cause intense pleuritic type pain with dyspnoea and often haemoptysis. ${ }^{4}$ On auscultation localised crackles and pleural rub may be heard.

The chest radiograph typically shows multiple nodular infiltrates scattered throughout both lung fields ${ }^{6}$ and small pleural effusions. Cavitation may already be detectable on the first chest radiograph. ${ }^{78}$ One of the striking features is the rapid progression of the lung lesions and pleural effusions, despite antibiotic therapy. Empyema develops in about 10\%$15 \%$ of cases. ${ }^{5}$

Considering the overall severity of the septicaemic illness, adult respiratory distress syndrome occurs in a relatively small proportion of cases and fewer than $10 \%$ of cases reported in cited literature since 1990 have required mechanical ventilation.

\section{Bone and joint manifestations}

In the antibiotic era, septic arthritis occurs in around 13\%$27 \%$ of cases (table 1). Although joint involvement may simply involve arthralgia, it typically progresses to a full blown culture positive arthritis. The pus may possess "a peculiarly foul odour". ${ }^{8}$ The hip is the most commonly infected joint in published series. Other joints reported to be involved include knee, shoulder, sacroiliac, elbow, and ankle.
Box 2: Suggestive features of Lemierre's

syndrome

- Previously fit adolescent or young adult.

- History of sore throat in preceding seven days.

- Onset of high fever and rigors.

- Signs of internal jugular venous thrombosis $130 \%-$ $40 \%)$.

- Dry cough and pleuritic chest pains.

- Chest radiograph shows multiple nodular lesions.

- Bilateral pleural effusions.

- Other features of metastatic abscess-for example, empyema or septic arthritis or skin/soft tissue abscess.

- Release of foul smelling pus from abscess or empyema.

Osteomyelitis is reported in fewer than 3\% of patients (table 1). Bones affected include humerus, ${ }^{22}$ femur, ${ }^{23}$ fibula, ${ }^{24}$ iliac bone, ${ }^{25}$ and cervical vertebra. ${ }^{8}$ In some cases the process is detected at an early stage but in others computed tomography revealed intraosseous gas with rapid destruction of bone. ${ }^{23}$

\section{Soft tissue lesions}

Skin and soft tissue lesions have been reported in $0 \%-16 \%$ of cases (table 1). Several reports have described abscesses developing in muscle, including gluteal ${ }^{46-29}$ and the abdominal wall. ${ }^{30}$ One report described the development of pyomyositis of the infraspinatus muscle. ${ }^{31}$

\section{Intra-abdominal sepsis}

Abnormal liver function is detected in $49 \%$ of patients ${ }^{5}$ and patients may be frankly jaundiced. ${ }^{6} 26{ }^{27} 32$ Liver abscesses, commonly multiple, ${ }^{83-35}$ and splenic abscesses ${ }^{25} 3637$ have both been described. Although abdominal pain is a common presenting symptom (eight out of 15 patients in Hagelskjaer's series $^{5}$ ), peritonitis is a rare complication. Hagelskjaer et al

Table 1 Major features of Lemierre's syndrome in published series

\begin{tabular}{|c|c|c|c|c|c|}
\hline & Sinave ef $a l^{6}$ & Eykyn $^{8}$ & Moreno $^{19}$ & $\begin{array}{l}\text { Leugers and } \\
\text { Clover }^{10}\end{array}$ & Hagelskjaer et $a l^{5}$ \\
\hline Number of cases & 38 & 29 & 11 & 39 & 15 \\
\hline Median age (range) & $20(2-38)$ & $22(16-27)$ & $18(13-23)$ & $18(9-38)$ & $18(14-31)$ \\
\hline $\begin{array}{l}F \text { necrophorum in blood } \\
\text { culture (\%) }\end{array}$ & 61 & 86 & 100 & 100 & 100 \\
\hline $\begin{array}{l}\text { Anaerobe in blood } \\
\text { culture (\%) }\end{array}$ & 97 & 100 & 100 & 100 & 100 \\
\hline $\begin{array}{l}\text { Other organisms in blood } \\
\text { culture (\%) }\end{array}$ & 50 & 3 & 9 & NR & NR \\
\hline IJV thrombosis (\%) & 26 & NR & 45 & 26 & NR \\
\hline Pulmonary lesions (\%) & 97 & 79 & 100 & 85 & 80 \\
\hline Septic arthritis (\%) & 13 & 14 & 18 & 26 & 27 \\
\hline Osteomyelitis (\%) & 2.6 & 3.0 & 9.0 & 2.5 & 0 \\
\hline $\begin{array}{l}\text { Skin and soft tissue } \\
\text { lesions (\%) }\end{array}$ & 16 & NR & NR & 5 & 0 \\
\hline Meningitis (\%) & 2.6 & 3.0 & NR & 2.5 & 0 \\
\hline Septic shock (\%) & 0 & NR & NR & 18 & NR \\
\hline Clinical jaundice (\%) & 11 & NR & NR & 49 & NR \\
\hline Renal failure (\%) & NR & 3 & 0 & 5 & NR \\
\hline Clinical DIC (\%) & 0 & 3.0 & 9.0 & 2.5 & 0 \\
\hline $\begin{array}{l}\text { Median duration of } \\
\text { fever after antibiotic } \\
\text { (days) }\end{array}$ & NR & NR & 10 & $8(2-16)$ & $12(1-20)$ \\
\hline Antibiotic duration & NR & $\begin{array}{l}\text { Average } \\
6 \text { weeks }\end{array}$ & $\begin{array}{l}\text { Minimum } \\
6 \text { weeks }\end{array}$ & 9-128 days & Median 18 days \\
\hline Mortality (\%) & 5.3 & 3.0 & 18.0 & 8.0 & 0 \\
\hline
\end{tabular}


suggested the pain might be caused by abdominal microabscesses.

\section{Central nervous system complications}

Purulent meningitis has been described in classical Lemierre's syndrome $e^{38-42}$ but is rare (table 1). This contrasts markedly with the relative frequency of meningitis in necrobacillosis secondary to otitis media or mastoiditis. ${ }^{584344}$ Cerebral abscess is a recognised but unusual complication. $^{825}{ }^{45}$ Abscesses can occur in various sites and may be multiple. ${ }^{25}$

A sinister development of internal jugular venous thrombosis is retrograde propagation to involve cranial sinuses including the cavernous sinus ${ }^{839}$ or sigmoid sinus. ${ }^{46}$

\section{Cardiovascular complications}

Despite the occurrence of persistent bacteraemia in patients with classical Lemierre's syndrome, endocarditis is exceptionally unusual. ${ }^{22}$ Occasional cases of pericarditis are reported. ${ }^{21}$ Similarly despite the severity and extent of the sepsis, septic shock requiring inotropic support is unusual (table 1).

\section{Renal complications}

For an infection associated with such severe sepsis, renal complications are remarkably uncommon. Acute renal failure requiring renal replacement therapy occurs in less than $5 \%$ of cases. ${ }^{8}{ }^{10}$ Case reports have described renal abscess, ${ }^{25}$ glomerulonephritis, ${ }^{47}$ and haemolytic uraemic syndrome. ${ }^{48}$

\section{Haematological complications}

Mild thrombocytopenia is not unusual. ${ }^{49}$ Laboratory evidence of disseminated intravascular coagulation (DIC) is relatively common, but clinically significant DIC is much less common $^{405051}$ being reported in $0 \%-9 \%$ in various series (table 1). DIC is occasionally severe enough to cause spontaneous bleeding ${ }^{50}$ and peripheral ischaemia and gangrene. ${ }^{51}$ One case of bilateral forefoot gangrene secondary to sepsis combined with effects of vasoconstrictor therapy was reported. $^{52}$

\section{DIFFERENTIAL DIAGNOSIS}

For a syndrome that is so characteristic, it is remarkable how often the diagnosis is missed until an anaerobic Gram negative rod is isolated from blood culture or other sterile site. There are several contributory factors. Thus many clinicians and even medical microbiologists have never seen a case and secondly the protean manifestations of the septic emboli can distract clinicians from the initial oropharyngeal sepsis. In addition the cases can present to a wide variety of specialties, including general medicine, otorhinolaryngology, orthopaedics, general surgery, and neurosurgery.

Many case reports describe how even at the stage of hospital admission, viral pharyngitis was the clinical

Box 3: Differential diagnosis of Lemierre's syndrome

- Viral pharyngitis.

- Infectious mononucleosis.

- Leptospirosis.

- Acute bacterial pneumonia.

- Atypical pneumonia.

- Aspiration pneumonia.

- Staphylococcal endocarditis/pneumonia.

- Intra-abdominal sepsis. diagnosis. The high C-reactive protein present in Lemierre's syndrome $^{16}$ should readily eliminate uncomplicated viral infection.

Infectious mononucleosis is often considered as the initial diagnosis and confusion can result from two factors. Firstly serologically confirmed Epstein-Barr virus infection may precede Lemierre's syndrome. ${ }^{1421}{ }^{40}$ In addition false positive heterophile antibody tests have been reported in patients with confirmed Lemierre's. ${ }^{14} 2124$ Distinguishing features will include the presence of generalised rather than purely cervical lymphadenopathy with infectious mononucleosis, unilateral signs of internal jugular venous thrombosis with Lemierre's syndrome, together with metastatic septic lesions and a markedly raised C-reactive protein. ${ }^{16}$

In the absence of frank abscesses, leptospirosis may be suggested by the presence of high fever, rigors, and abnormal liver function. ${ }^{3}$

The most obvious clinical and radiological feature is the rapidly progressive lung lesion and not infrequently patients are thought to have acute bacterial pneumonia, Legionnaire's disease, ${ }^{16}$ or aspiration pneumonia. ${ }^{19}$ For those with access to molecular diagnostic methods further confusion can apparently result from false positive mycoplasma polymerase chain reaction tests..$^{53}$ The key distinguishing features are the fact that lung involvement in Lemierre's syndrome is preceded by sore throat, may be accompanied by internal jugular vein thrombosis, and that initially lung lesions consist of multiple diffusely disseminated nodular lesions which rapidly cavitate.

The presence of rapidly developing cavitating lung lesions is often mistaken for staphylococcal pneumonia or right sided staphylococcal endocarditis. Leugers and Clover reported a patient with cavitating lung lesions in whom Staphylococcus aureus was isolated from sputum before blood cultures grew $F$ necrophorum. ${ }^{10}$ Among the series of Moreno et al of 11 cases, six were initially treated with antistaphylococcal therapy for presumed right sided endocarditis. ${ }^{19}$ Similarly four of five of Seidenfield et al's cases were treated initially for presumed staphylococcal infection. ${ }^{18}$

The fact that patients frequently present with abdominal pain and have liver function abnormalities can often mislead clinicians into suspecting intra-abdominal sepsis. This can be compounded if an initial isolation of an anaerobic Gram negative rod is assumed to be Bacteroides $s p .{ }^{16}$

In patients in whom drainage of an abscess or empyema is undertaken before the diagnosis has been made, the foul smelling pus may be a crucial clue:

"The diagnosis of this infection may be suggested by the peculiar odour-like Limburger or overripe Camembert cheese- of pus produced by it". ${ }^{3}$

\section{Radiological diagnosis (figs 2-4)}

Internal jugular venous thrombophlebitis can only be confirmed by imaging techniques. Ultrasonography is often used as the initial modality for demonstration of internal jugular vein thrombosis, being less expensive and not requiring exposure to radiation. However it provides poor imaging beneath the clavicle and mandible and can miss a fresh thrombus with low echogenicity. ${ }^{54}$ High resolution computed tomography has a higher sensitivity but clearly involves exposure to radiation. ${ }^{55}$ Magnetic resonance imaging has been used successfully to identify thrombus not detected on computed tomography but is much more expensive. ${ }^{56} 57$ These techniques can also distinguish between localised abscess formation in the neck and internal jugular vein thrombophlebitis, thus potentially avoiding unnecessary 


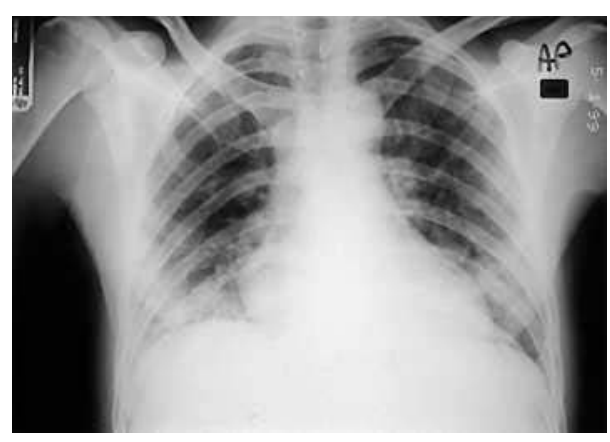

Figure 2 Chest radiograph of 35 year old man with Lemierre's syndrome showing bilateral opacification particularly in right lower zones.

surgery. ${ }^{56}$ Localisation of abscesses requiring drainage is greatly assisted. ${ }^{58}$

Septic emboli in the lungs may produce the characteristic radiographic appearance of multiple peripheral round and wedge shaped opacities that rapidly progress to cavitation. ${ }^{59}$ However in other patients initial radiography may be clear or show non-specific patchy consolidation suggestive of bronchopneumonia. Computed tomography of the chest often reveals diagnostic information with characteristic appearances of septic infarcts-that is, predominantly peripheral nodules showing cavitation. ${ }^{55}$ With administration of contrast, the lesions often show peripheral enhancement with central areas of reduced attenuation. The so-called "feeding vessel" sign is also characteristic of septic pulmonary embolism. ${ }^{55}$

\section{Laboratory diagnosis}

Basic biochemical and haematological data provide some pointers. Patient typically have a neutrophil leucocytosis, liver function tests are abnormal in approximately $50 \%$ of patients ${ }^{5}$ and the C-reactive protein is invariably raised. ${ }^{16}$ However the key to laboratory confirmation of the diagnosis of Lemierre's syndrome is culture of appropriate specimens among which blood cultures are at the forefront. It is also crucial to culture pus drained from any site, including localised abscesses in the neck, empyema, septic arthritis, bone, and soft tissue abscesses.

Anaerobic culture media and techniques are now well established and routine in laboratories, but it must be recognised that $F$ necrophorum takes at least 48 hours, and sometimes up to seven days to grow in blood cultures. In

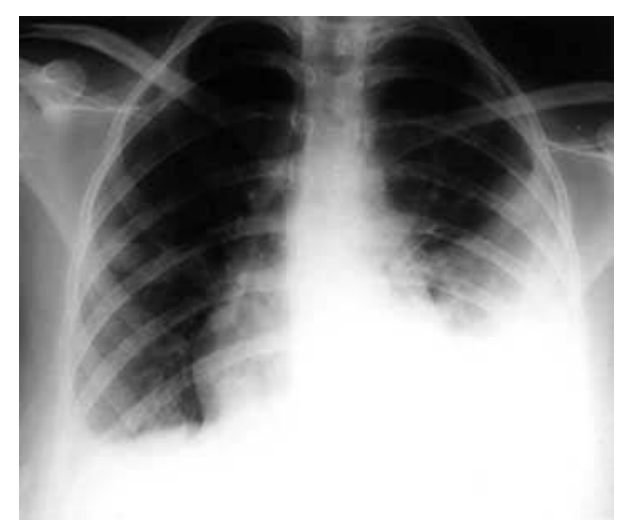

Figure 3 Chest radiograph of 16 year old girl with Lemierre's syndrome confirmed by culture. There is a substantial effusion in the left lower zone and several focal lesions in the peripheral lung fields. Drainage of the effusions yielded foul smelling pus.

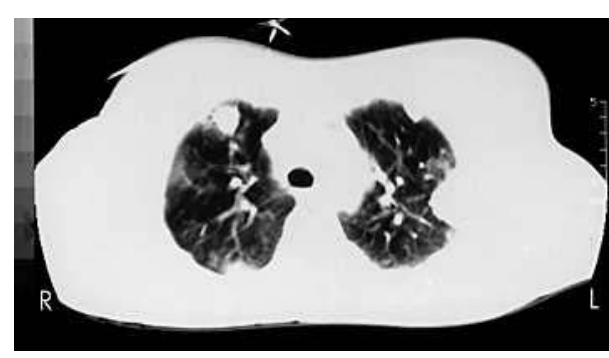

Figure 4 Computed tomogram of same patient as in fig 3 showing several peripherally located lesions, some of which are intraparenchymal and some are pleurally based.

addition the isolation of a non-sporing anaerobic Gram negative rod in a specimen may readily be assumed to be a Bacteroides $s p$. This may lead to erroneous suppositions as to the source of infection and thus to inappropriate investigations. It is essential that all anaerobic Gram negative rods from sterile sites are identified to at least genus level and that fusobacteria are identified to species level. The initial clue to the identity should come from the morphology of the organism on a Gram stained smear. This typically shows Gram negative filaments (with rounded, not tapered ends), of varying length and with coiling and irregular swelling of the cell. ${ }^{3}$

\section{THERAPY}

On first encountering the condition one is bound to be struck by the slow response to antibiotics. In three series the median time from initiation of appropriate antibiotic therapy to resolution of fever ranged from eight to 12 days (table 1 ).

Several factors may account for the slow response. Firstly there may be overt collections of pus in sites not amenable to drainage such as lung and liver. Secondly the nature of the pathogenic process with septic thrombophlebitis means that the organism may be sequestered in a site where antibiotic penetration is poor. The third factor is antibiotic resistance. $F$ necrophorum is intrinsically resistant to gentamicin and quinolones have relatively poor activity. In vitro erythromycin resistance has been documented in up to $22 \%$ of cases. ${ }^{13}$ Brazier et al found $2 \%$ of isolates to be penicillin resistant, ${ }^{12}$ whereas Applebaum et al reported $22 \%$ of their isolates to be $\beta$-lactamase producers. ${ }^{60} \mathrm{~F}$ necrophorum remains generally sensitive to clindamycin and metronidazole. ${ }^{13}$

In a rare condition efficacy data are inevitably somewhat anecdotal. If the diagnosis is not recognised, patients may be treated for atypical pneumonia or staphylococcal endocarditis/pneumonia. Quinolones, macrolides, and antistaphylococcal therapy with agents such as flucloxacillin are considered inadequate therapy for Lemierre's syndrome. There is a general impression that despite in vitro sensitivity, penicillin is relatively ineffective. ${ }^{57}$ Some data suggest clindamycin is more effective in therapy of lung abscess. ${ }^{61}$ However the available data suggest that metronidazole may be associated with the most rapid response. Barker et al suggest that this may be due to better tissue penetration than penicillin. ${ }^{62}$ Moore-Gillon et al commented: "despite a lack of conclusive evidence our clinical impression is that definitive improvement coincided with introduction of metronidazole in at least 3 of our patients". ${ }^{7}$

Because of the frequent occurrence of mixed infection, monotherapy with metronidazole is considered to be inadvisable and penicillin and metronidazole is commonly recommended. ${ }^{8}$ No general figure for duration of therapy can be recommended since so many factors affect the response to therapy. Many patients are treated for four weeks, although, 


\section{Box 4: Management of Lemierre's syndrome}

- Antibiotic therapy including metronidazole.

- Drainage of accessible abscesses.

- Anticoagulation is rarely indicated.

- Internal jugular vein ligation/excision is rarely indicated.

given metronidazole's good oral availability, a switch to oral therapy can be made when the patient is afebrile.

The second element of management is the identification of collections of pus amenable to drainage. This may include local lesions in the neck including parapharyngeal and peritonsillar abscesses, empyema, septic arthritis, cerebral abscess, and osteomyelitis. ${ }^{10} 11$

The use of anticoagulants has been controversial. Armstrong et al commented "Only a minority of patients receive anticoagulation in most studies and yet do well". ${ }^{57}$ With vigorous antibiotic therapy and surgical drainage, anticoagulation should probably be reserved for patients with evidence of retrograde progression to the cavernous sinus. ${ }^{11}$

A more drastic form of therapy that has been employed is ligation or resection of the internal jugular vein. This is probably only indicated in patients with persistent septic embolisation despite antibiotics. ${ }^{63}$

In the preantibiotic era the prognosis of Lemierre's syndrome was grave. Lemierre reported that 18 of 20 of his cases died. ${ }^{4}$ With antibiotic therapy, despite the severity of sepsis, recovery is normal with mortality figures in series ranging from $0 \%$ to $18 \%$ (table 1 ).

The illness varies in its severity. Harar et al reported two of three of their cases being discharged from hospital within four days ${ }^{64}$ whereas Vohra described a fulminating, ultimately fatal, case with abscesses in multiple organs. ${ }^{25}$ There is some evidence that delay in initiation of antibiotics affects outcome. Thus Leugers and Clover found that two deaths occurred in eight patients in whom antibiotic therapy was delayed for four days or more, contrasting with only one death in 29 patients who were promptly treated. ${ }^{10}$

\section{PREVENTION}

As described above a number of papers have suggested a recent resurgence of Lemierre's, which has been putatively linked to the pressure to avoid prescription of antibiotics for sore throat.

The clinical dilemma is obvious. On the one hand widespread prescription of antibiotics inevitably means that many patients with viral infections are unnecessarily treated, with the dual risk of drug side effects to the individual and fostering of the rise in antibiotic resistant bacteria. ${ }^{13}$ On the other hand not only do antibiotics shorten the duration of symptoms with bacterial sore throat but also can prevent suppurative complications such as peritonsillar abscess. ${ }^{65}$

\section{Box 5: Features suggesting bacterial cause for} pharyngitis

- High fever particularly with rigors.

- Absence of accompanying coryzal symptoms.

- Tender cervical lymphadenopathy.

- Severe sore throat persists $>3$ days.
Most studies relate to streptococcal infection. In the case of Lemierre's syndrome there is no doubt that low dose oral antibiotics will have little impact once the process of septic thrombophlebitis spreading from the tonsillar veins has commenced. However it is possible that therapy at an earlier stage could be effective.

"If we physicians are going to reserve antibiotic use in head and neck infections we must be aware of the lessons the pre-antibiotic era taught us. Lemierre's syndrome should be remembered as a deadly but preventable complication of pharyngitis".

The dilemma for general practitioners is highlighted in an editorial titled "the dangerous needle in the haystack of sore throats". ${ }^{66}$ There is no sure way of detecting all patients at risk of suppurative complications but we would suggest that patients presenting with the features in box 5 should have blood taken for infectious mononucleosis screening and $\mathrm{C}$-reactive protein and should be commenced on antibiotic therapy if heterophile antibody tests are negative.

\section{ACKNOWLEDGEMENTS}

We would like to thank Dr Clive Hamilton-Wood for advice on the radiographic images.

\section{Authors' affiliations}

T Riordan, M Wilson, Microbiology Department, Royal Devon and Exeter Healthcare NHS Trust, Exeter, UK

\section{REFERENCES}

1 Langworth BF. Fusobacterium necrophorum. Its characteristics and role as an animal pathogen. Bacteriol Rev 1977:41:373-90.

2 Courmont $P$, Cade A. Sur une septico-pyohemie de l'homme simulant la peste et causée par un streptobacille anaerobie. Arch Med Exp Anat Pathol $1900 ; 12: 393-418$

3 Alston JM. Necrobacillosis in Great Britain. BMJ 1955;ii:1524-8.

4 Lemierre A. Septicaemias and anaerobic organisms. Lancet 1936;i:701-3.

5 Hagelskjaer LH, Prag J, Malczynski J, et al. Incidence and clinical epidemiology of necrobacillosis, including Lemierre's syndrome, in Denmark 1990-1995. Eur J Clin Microbiol Infect Dis 1998;17:561-5.

6 Sinave CP, Hardy GJ, Fardy PW. The Lemierre syndrome: suppurative thrombophlebitis of the internal jugular vein secondary to oropharyngeal infection. Medicine (Baltimore) 1989;68:85-94.

7 Moore-Gillon J, Lee TH, Eykyn SJ, et al. Necrobacillosis: a forgotten disease. BMJ 1984:288:1526-7.

8 Eykyn SJ. Necrobacillosis. Scand J Infect Dis Suppl 1989;62:41-6.

9 Jones JW, Riordan T, Morgan MS. Investigation of postanginal sepsis and Lemierre's syndrome in the South West Peninsula. Commun Dis Public Health $2001: 4: 278-81$.

10 Leugers CM, Clover R. Lemierre syndrome: postanginal sepsis. J Am Board Fam Pract 1995;8:384-91.

11 Lustig LR, Cusick BC, Cheung SW, et al. Lemierre's syndrome: two cases of postanginal sepsis. Otolaryngol Head Neck Surg 1995; 1 12:767-72.

12 Brazier JS, Hall V, Yusuf E, et al. Fusobacterium necrophorum infections in England and Wales 1990-2000. J Med Microbiol 2002;51:269-72.

13 Standing Medical Advisory Committee. The path of least resistance. London: Department of Health, 1998.

14 Dagan R, Powell KR. Postanginal sepsis following infectious mononucleosis. Arch Intern Med 1987; 147:1581-3.

15 Roberts DS. The pathogenic synergy of $F$ necrophorum and $C$ pyogenes. Influence of leucocidal extoxin of $\mathrm{F}$ necrophorum. $\mathrm{Br} J$ Exp Pathol 1967:48:665-73.

16 Hagelskjaer LH, Kristensen L, Prag J. Human necrobacillosis, with emphasis on Lemierre's syndrome. Clin Infect Dis 2000;31:524-32.

17 Hall C. Sepsis following pharyngeal infections. Ann Otol Rhinol Laryngol 1939;48:905-25.

18 Seidenfeld SM, Sutker WL, Luby JP. Fusobacterium necrophorum septicemia following oropharyngeal infection. JAMA 1982;248:1348-50.

19 Moreno S, Garcia Altozano J, Pinilla B, et al. Lemierre's disease: postanginal bacteremia and pulmonary involvement caused by Fusobacterium necrophorum. Rev Infect Dis 1989;1 1:319-24.

20 Cosgrove EF, Colodny SM, Pesce RR. Adult respiratory distress syndrome as a complication of postanginal sepsis. Chest 1993;103:1628-9.

21 Moller K, Dreijer B. Postanginal sepsis (Lemierre's disease): a persisten challenge. Presentation of 4 cases. Scand J Infect Dis 1997;29:191-4.

22 Epstein M, Pearson AD, Hudson SJ, et al. Necrobacillosis with pancytopenia. Arch Dis Child 1992;67:958-9. 
23 Foulkes GD, Johnson CE, Katner HP. Fusobacterium osteomyelitis associated with intraosseous gas. Clin Orthop 1990;251:246-8.

24 Stahlman GC, DeBoer DK, Green NE. Fusobacterium osteomyelitis and pyarthrosis: a classic case of Lemierre's syndrome. J Pediatr Orthop 1996; 16:529-32

25 Vohra A, Saiz E, Ratzan KR. A young woman with a sore throat, septicaemia and respiratory failure. Lancet 1997;350:928

26 Koay CB, Heyworth T, Burden P. Lemierre syndrome-a forgotten complication of acute tonsillitis. J Laryngol Otol 1995;109:657-61.

27 Lee BK, Lopez F, Genovese M, et al. Lemierre's syndrome. South Med 1997;90:640-3.

28 Pickering MC, Barkham T, Mason JC, et al. Bilateral gluteal abscesses as a unique manifestation of fusobacterium septicaemia. Rheumatology (Oxford) 2000;39:224-5.

29 Abele-Horn M, Emmerling P, Mann JF. Lemierre's syndrome with spondylitis and pulmonary and gluteal abscesses associated with Mycoplasma pneumoniae pneumonia. Eur J Clin Microbiol Infect Dis 2001;20:263-6.

30 Martin MJ, Wright ED. A case of Fusobacterium necrophorum sepsis. J Infect 1995;31:151-2.

31 Wolf RF, Konings JG, Prins TR, et al. Fusobacterium pyomyositis of the shoulder after tonsillitis. Report of a case of Lemierre's syndrome. Acta Orthop Scand 1991;62:596-6.

32 Ray J, O'Gradaigh D, Tighe R. A young man with a characteristic syndrome. Postgrad Med J 2000;76:51-2

33 Embree JE, Williams T, Law BJ. Hepatic abscesses in a child caused by Fusobacterium necrophorum. Pediatr Infect Dis J 1988;7:359-60.

34 Hagelskjaer L, Pedersen G. Fusobacterium necrophorum septicemia complicated by liver abscess. A case report. APMIS 1993;101:904-6.

35 Katzenstein TL, Jensen T, Arpi M. Multiple liver abscesses caused by Fusobacterium necrophorum infection. Ugeskr Laeger 1994;156:6706-7.

36 Hwang JJ, Lau YJ, Hu BS, et al. Haemophilus parainfluenzae and Fusobacterium necrophorum liver abscess: a case report. J Microbiol Immunol Infect 2002;35:65-7.

37 Kern W, Doldere $M$, Krieger D, et al. Lemierre's syndrome with splenic abscesses. Dtsch Med Wochenschr 1992;117:1513-7.

38 O'Grady LR, Ralph ED. Anaerobic meningitis and bacteremia cause by fusobacterium species. Am J Dis Child 1976;130:871-3.

39 Jones TH, Bergvall V, Bradshaw JP. Carotid artery stenoses and thrombosis secondary to cavernous sinus thromboses in Fusobacterium necrophorum meningitis. Postgrad Med J 1990;66:747-50.

40 Busch N, Mertens PR, Schonfelder T, et al. Lemierre's post-tonsillitis sepsis with meningitis and intravascular consumption coagulopathy as complication of infectious mononucleosis with pansinusitis. Dtsch Med Wochenschr 1996;121:94-8.

41 Sherlock R, Le Saux N. Your diagnosis please. 15 year old girl with fever, headache and vomiting. Pediatr Infect Dis J 2002;21:439.

42 Bach MC, Roediger JH, Rinder HM. Septic anaerobic jugular phlebitis with pulmonary embolism: problems in management. Rev Infect Dis 1988;10:424-7.

43 Reynolds MA, Hart CA, Harris F, et al. Anaerobes in acute otitis media. J Infect $1985 ; 10: 262-4$.
44 Tarnvik A Sundqvist G, Gothefors L, et al. Meningitis caused by Fusobacterium necrophorum. Eur J Clin Microbiol 1986;5:353-5.

45 Meis JF, Polder TW, van de Kar P, et al. Multiple brain abscesses and bacteremia in a child due to Fusobacterium necrophorum. Infection 1993;21:174-6.

46 Agarwal R, Arunachalam PS, Bosman DA. Lemierre's syndrome: a complication of acute oropharyngitis. J Laryngol Otol 2000;1 14:545-7

47 Carrie S, Fenton PA. Necrobacillosis - an unusual case of pharyngotonsillitis. $J$ Laryngol Otol 1994; 108:1097-8.

48 Chand DH, Brady RC, Bissler JJ. Hemolytic uremic syndrome in an adolescent with Fusobacterium necrophorum bacteremia. Am J Kidney Dis 2001;37:E22.

49 Ellis GR, Gozzard DI, Looker DN, et al. Postanginal septicaemia (Lemierre's disease) complicated by haemophagocytosis. J Infect 1998;36:340-1.

50 Potter MN, Drysdale HC, Price PA, et al. Disseminated intravascular coagulation with Fusobacterium necrophorum septicaemia. Postgrad Med J 1988;64:155-6.

51 Page Y, Comtet C, Tardy B, et al. Disseminated intravascular coagulation in Fusobacterium necrophorum septicemia. Scand J Infect Dis 1990;22:743-7.

52 Soave RL, Kuchar DJ. Bilateral forefoot gangrene secondary to Lemierre's disease. J Am Podiatr Med Assoc 2001:91:147-9.

53 Jensen JS, Bruun B, Gahrn-Hansen B. Unexpected cross-reaction with Fusobacterium necrophorum in a PCR for detection of mycoplasmas. J Clin Microbiol 1999;37:828-9

54 Golpe R, Marin B, Alonso M. Lemierre's syndrome (necrobacillosis). Postgrad Med J 1999:75:141-4

55 Screaton NJ, Ravenel JG, Lehner PJ, et al. Lemierre's syndrome: forgotten but not extinct-report on four cases. Radiology 1999;213:369-74.

56 Auber AE, Mancuso PA. Lemierre's syndrome: magnetic resonance imaging and computed tomographic appearance. Mil Med 2000;165:638-40.

57 Armstrong AW, Spooner K, Sanders JW. Lemierre's syndrome. Curr Infect Dis Rep 2000;2:168-73.

58 Carlson ER, Bergamo DF, Coccia CT. Lemierre's syndrome: two cases of a forgotten disease. J Oral Maxillofac Surg 1994;52:74-8.

59 Gudinchet F, Maeden P, Neveceral P, et al. Lemierre's syndrome in children. Chest 1997; 112:271-3.

60 Applebaum PC, Spingler SJ, Jacobs MR. $\beta$ lactamase production and sensitivities to amoxycillin, amox-clavulanate. Antimicrob Agents Chemother 1990;34:1546-50.

61 Levison ME, Mangura CT, Lorber B, et al. Penicillin versus clindamycin for the treatment of anaerobic lung abscess. Ann Intern Med 1983;98:466-71.

62 Barker J, Winer-Muram HT, Grey SW, et al. Lemierre syndrome. South Med J 1996;89:1021-3.

63 Venkatarama MT, Polica M. Fever sore throat and pulmonary infiltrates in a 20 year old man. Chest 1997;112:268-70.

64 Harar RP, Macdonald A, Pullen D, et al. Lemierre's syndrome: are we missing this life threatening infection? J Otorhinolaryngol Relat Spec 1996:58:178-81.

65 Zwart S, Sachs APE, Ruijs GJHM, et al. Penicillin for acute sore throat: randomised double blind trial of seven days versus three days treatment or placebo in adults. BMJ 2000;320:150-4.

66 Gant V. The dangerous needle in the haystack of sore throats. Hospital Medicine 2000;1:376-7. 